\title{
Cement pavement performance evaluation based on the discrete Hopfield neural network
}

\author{
Huan Liu ${ }^{1 *}$, Peng Liu², Qiuyu Peng ${ }^{1}$ \\ ${ }^{1}$ Chang'an University, Xi'an, Shaanxi, 710064, China \\ ${ }^{2}$ China Communication South Road and Bridge CO., LTD, Beijing, 101149, China
}

\begin{abstract}
Because of the deficiency of the index of cement pavement performance evaluation and the defect of the evaluation method in the specification, the performance of the pavement is comprehensively evaluated by seven optimized indexes and grading standards that reflect functional performance and structure of the pavement. Because the discrete Hopfield neural network is available with simple construction procedure, less training samples, and strong objectivity. The DHNN is constructed by MATLAB to evaluate the performance of test pavement. The ideal cement pavement performance grading evaluation index matrix and 6 places unclassified of test pavement performance evaluation index matrix are input to the neural network then the evaluation result is obtained after simulating and learning. Finally, comparing the result of the DHNN with the fuzzy complex matter element method and the nonlinear fuzzy method, it is proved that the discrete Hopfield neural network evaluation method is reliable.
\end{abstract}

\section{Introduction}

Pavement performance evaluation plays an important role in the road maintenance and maintenance process. In the cement pavement maintenance plan decision system, cement pavement performance evaluation is closely related to predicting pavement performance, developing maintenance plans, allocating maintenance funds, and adopting maintenance measures. Therefore, accurate and effective cement pavement performance evaluation methods are of great significance for the long-term planning and management of maintenance work.

Cement pavement performance evaluation are roughly divided into two categories. The first category is the evaluation of pavement performance according to relevant normative standards. The road surface damage condition, structural bearing capacity, road surface driving quality and anti-sliding ability are used to evaluate the performance of cement pavement in the "Technical Specification for Highway Cement Concrete Pavement Maintenance" (JTJ 073.1-2001). However, the evaluation index of the bearing capacity of the surface layer lacks the grading standard and does not give the evaluation index of the carrying capacity of the grassroots ${ }^{[1]}$. Besides, multiple independent individual indicators are used to evaluate road conditions and lacks a comprehensive evaluation indicator. Different cement pavement conditions are difficult to compare. It is inconvenient for the maintenance department to coordinate the overall situation, planning management and other work. The performance of a certain aspect of the cement pavement directly determines the maintenance measures to be taken.
The situation that is prone to cause "small problems, big conservation" was created. The above phenomenon maintenance and maintenance program decision-making and fund allocation are very unfavourable. The pavement performance index PQI value was used to evaluate the performance of the cement pavement in the "Technical Evaluation Standard for Highway Technical Conditions" (JTG H20-2007). In the actual maintenance process, the bearing capacity of the pavement structure and the void rate at the bottom of the board are also the main factors affecting the performance of the pavement. The low bearing capacity of the structure is likely to cause the pavement to sink and sink. The concentrated stress at the bottom of the plate is likely to cause the plate to be staggered when the load is applied to the vacant plate ${ }^{[2]}$. Therefore, the evaluation index of cement pavement performance in "Standard" is insufficient and the determination of index weight is subjective, which is easy to produce a phenomenon that the evaluation result is inconsistent with the actual condition of the road surface.

The second category is the comprehensive evaluation of the performance of cement pavement using mathematical methods, which is based on the optimization index and grading standard of cement pavement performance evaluation. This type of method largely compensates for the shortcomings of the standard evaluation method. It supports basic algorithms such as vector machine and fuzzy recognition, and many comprehensive evaluation methods for its optimization and improvement. At the same time, it has a specific SVM classification algorithm ${ }^{[3]}$, a fuzzy hierarchical synthesis method ${ }^{[4]}$ and a fuzzy complex matter element method ${ }^{[5]}$ and a nonlinear fuzzy method ${ }^{[6]}$ applied according to the 
improved "norm" evaluation index. However the above algorithms have their own shortcomings. For example, the fuzzy level synthesis method cannot avoid the shortcomings of FCE-AHP. The nonlinear fuzzy method still has problems such as subjectivity.

The performance evaluation method of cement pavement was studied based on the optimization index and grading standard for evaluating the performance of cement pavement proposed in the literature. The performance indicators of cement pavement are obtained by testing and testing. However, due to high test cost, complicated and cumbersome test process, the sampling points are small and the sample data is insufficient. That has brought many difficulties to accurately evaluate the performance of cement pavement. Therefore, this paper proposes a comprehensive evaluation of cement pavement performance using discrete Hopfield neural network method. Compared with the traditional method, this method does not require a large number of training samples and has a lot of objectivity. Based on the test data of a test section, the discrete Hopfield neural network method was applied to evaluate the performance of cement pavement. Compared with the fuzzy complex element method and the nonlinear fuzzy method, the discrete Hopfield neural network method is proved to be reliable.

\section{The basic principle of discrete Hopfield neural network}

Hopfield neural network was proposed by American physicist J.J Hopfield in 1982. Hopfield neural network and learning algorithm is a binary neural network. The output of neurons is only 1 and -1 , indicating that neurons are in an activated and suppressed state, respectively. So it is also called Discrete Hopfield Neural Network (DHNN).

\subsection{Discrete Hopfield neural network structure}

Some tex DHNN has the characteristics of single layer and binary. For example, a discrete Hopfield neural network structure is composed of three neurons as shown in FIG. Then layer 0 is only an input to the network, it does not represent the actual neurons. The first layer is a neuron that performs summation of the input information and the weight coefficient product, and is processed by the nonlinear function $\mathrm{f}$ to generate output information. $\mathrm{f}$ is a simple threshold function. If the output information of the neuron is greater than the threshold $\theta$, the output of the neuron takes a value of 1 . If the output information of the neuron is less than the threshold $\theta$, then the output of the neuron takes the value $-1^{[7]}$.

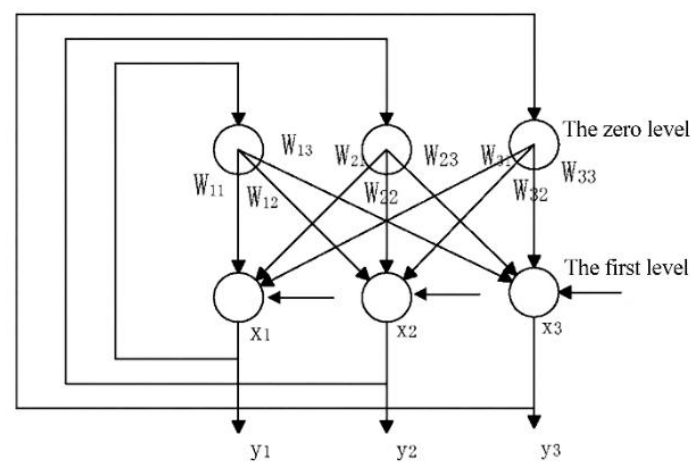

Figure 1. Structure of discrete Hopfield neural network

\subsection{Design of weight coefficient matrix for discrete Hopfield neural network}

The purpose of the DHNN weight coefficient matrix design is to ensure that the stability of the system during asynchronous operation and the stable equilibrium point of all memory requirements can converge. The two most common design methods are. External product method and orthogonal method. The steps designed by the outer product method are as follows:

Step1: According to the sample vector $t=\left[t^{l}, t^{2}, \ldots, t^{N}\right]$. If the state of $t^{k}$ is 1 or -1 , the weight coefficient matrix was calculated by the "outer product rule" $\boldsymbol{W}$;

$$
\boldsymbol{W}=\sum_{k=1}^{N}\left[t^{k}\left(t^{k}\right)^{T}-\boldsymbol{I}\right]
$$

Step2: The initial value of the network output $y_{i}(0)=p_{i}(i=1,2, \ldots, n)$ is the test sample $p_{i}(i=1,2, \ldots, n)$. Then setting the number of iterations;

Step3: Perform an iterative calculation. The formula is:

$$
y_{i}(k+1)=f\left(\sum_{j=1}^{N} w_{i j} y_{j}\right)
$$

Step4: Terminate the iteration when the maximum number of iterations or the neuron output state remains unchanged, otherwise return to Step3 to continue the iteration.

The steps of the orthogonalization method are as follows:

Step1: Input $N$ modes $t=\left[t^{1}, t^{2}, \ldots, t^{N}\right]$ and parameters $\tau, h$;

Step2: Calculate $\boldsymbol{A}=\left[t^{1}-t^{N}, t^{2}-t^{N}, \ldots, t^{N-1}-t^{N}\right]$;

Step3: Do singular value decomposition $\boldsymbol{A}=\mathrm{USVT}$ for $\boldsymbol{A}$, and calculate the $\operatorname{rank} k=\operatorname{rank}(\boldsymbol{A})$ of $\boldsymbol{A}$;

Step4: Calculate $\boldsymbol{T}^{P}$ and $\boldsymbol{T}^{m}$ from $\boldsymbol{U}^{P}=\left[\mathrm{U}^{1}, \mathrm{U}^{2}, \ldots, \mathrm{U}^{\mathrm{k}}\right]$ and $\boldsymbol{U}^{m}=\left[U^{k+1}, U^{k+2}, \ldots, U^{N}\right]$ respectively:

$$
T^{p}=\sum_{i=1}^{k} U^{i}\left(U^{i}\right)^{T}, T^{m}=\sum_{i=k+1}^{N} U^{i}\left(U^{i}\right)^{T}
$$

Step5: Calculate $\boldsymbol{W}^{t}=\boldsymbol{T}^{P}-\tau \times \boldsymbol{T}^{n}, \boldsymbol{b}^{t}=\boldsymbol{t}^{N}-\boldsymbol{W}^{t} \times \boldsymbol{t}^{N}$;

Step6: Calculate $\boldsymbol{W}=\exp \left(h \times \boldsymbol{W}^{t}\right)$;

Step7: Calculate $\boldsymbol{b}$ :

$$
\boldsymbol{b}=\boldsymbol{U} \times\left[\begin{array}{cc}
C_{1} \times \boldsymbol{I}(k) & 0(k, N-k) \\
0(N-k, k) & C_{2} \times \boldsymbol{I}(N-k)
\end{array}\right] \times \boldsymbol{U}^{T} \times \boldsymbol{b}^{t}
$$

Where:

$$
C_{1}=\exp (h)-1, C_{2}=-[\exp (-\tau \times h)-1] / \tau
$$




\section{Performance Evaluation of Cement Pavement Based on DHNN}

The essence of discrete Hopfield neural network classification learning is the process of gradually approaching the evaluation index of typical classification level to the equilibrium point of DHNN. Hopfield neural network was proposed by American physicist J.J Hopfield in 1982. Hopfield neural network and learning algorithm is a binary neural network. The output of neurons is only
1 and -1 , indicating that neurons are in an activated and suppressed state, respectively. So it is also called Discrete Hopfield Neural Network (DHNN).

\subsection{Ideal cement pavement performance grade evaluation index matrix}

The grading standards for cement pavement performance evaluation are shown in Table 1:

Table 1. Cement pavement performance grading standard

\begin{tabular}{cccccccc}
\hline \multirow{2}{*}{ Grade } & \multicolumn{3}{c}{ Pavement performance } & \multicolumn{4}{c}{ Pavement structure } \\
\cline { 2 - 7 } & PCI & RQI & IFI & DBL/\% & Et/MPa & Kj/\% & Rv/\% \\
\hline Excellent & $100 \sim 70$ & $10.0 \sim 7.0$ & $0.322 \sim 0.257$ & $0 \sim 5$ & $>295$ & $>80$ & $0 \sim 5$ \\
Good & $69 \sim 55$ & $6.9 \sim 4.5$ & $0.256 \sim 0.154$ & $6 \sim 10$ & $265 \sim 295$ & $56 \sim 80$ & $6 \sim 10$ \\
Medium & $54 \sim 40$ & $4.4 \sim 2.0$ & $0.153 \sim 0.012$ & $11 \sim 20$ & $215 \sim 264$ & $31 \sim 55$ & $11 \sim 20$ \\
Poor & $39 \sim 20$ & $1.9 \sim 0$ & $0.011 \sim 0$ & $21 \sim 40$ & $0 \sim 215$ & $30 \sim 0$ & $21 \sim 40$ \\
\hline
\end{tabular}

Discrete Hopfield neural network neuron output states are only 1 and -1 . When the evaluation index is mapped to the neuron state, the corresponding cement state is 1 when the cement pavement performance index is greater than or

$$
\left[\begin{array}{rrrr}
1 & -1 & -1 & -1 \\
1 & -1 & -1 & -1 \\
1 & -1 & -1 & -1 \\
1 & -1 & -1 & -1 \\
1 & -1 & -1 & -1 \\
1 & -1 & -1 & -1 \\
1 & -1 & -1 & -1
\end{array}\right]\left[\begin{array}{llll}
-1 & 1 & -1 & -1 \\
-1 & 1 & -1 & -1 \\
-1 & 1 & -1 & -1 \\
-1 & 1 & -1 & -1 \\
-1 & 1 & -1 & -1 \\
-1 & 1 & -1 & -1 \\
-1 & 1 & -1 & -1
\end{array}\right]\left[\begin{array}{llll}
-1 & -1 & 1 & -1 \\
-1 & -1 & 1 & -1 \\
-1 & -1 & 1 & -1 \\
-1 & -1 & 1 & -1 \\
-1 & -1 & 1 & -1 \\
-1 & -1 & 1 & -1 \\
-1 & -1 & 1 & -1
\end{array}\right]\left[\begin{array}{llll}
-1 & -1 & -1 & 1 \\
-1 & -1 & -1 & 1 \\
-1 & -1 & -1 & 1 \\
-1 & -1 & -1 & 1 \\
-1 & -1 & -1 & 1 \\
-1 & -1 & -1 & 1 \\
-1 & -1 & -1 & 1
\end{array}\right]
$$

to a certain level, and vice versa is -1 . The ideal grade evaluation index matrix for evaluating cement pavement performance is as follows:

\subsection{Grade evaluation indicator matrix of the unclassified cement pavement performance}

The DHNN evaluation method was applied to the performance evaluation of concrete engineering cement pavement. The measured data of cement pavement performance at $6(\mathrm{~A}, \mathrm{~B}, \mathrm{C}, \mathrm{D}, \mathrm{E}, \mathrm{F})$ of a road test section are shown in Table 2:

Table 2. Test values of evaluation indexes

\begin{tabular}{cccccccc}
\hline Test road & PCI & RQI & IFI & DBL $/ \%$ & Et $/ \mathrm{MPa}$ & $\mathrm{Kj} / \%$ & $\mathrm{Rv} / \%$ \\
\hline 1 & 61 & 4.7 & 0.156 & 8 & 304 & 44 & 18 \\
2 & 18 & 2.0 & 0.015 & 25 & 220 & 35 & 21 \\
3 & 82 & 6.1 & 0.258 & 6 & 310 & 82 & 12 \\
4 & 48 & 1.8 & 0.155 & 20 & 218 & 36 & 25 \\
5 & 22 & 2.3 & 0.146 & 35 & 190 & 25 & 10 \\
6 & 56 & 4.6 & 0.153 & 15 & 292 & 60 & 15 \\
\hline
\end{tabular}

Compare the measured values with Table1.Six unclassified cement pavement performance grade evaluation indicator matrices were obtained: 


$$
\begin{aligned}
& {\left[\begin{array}{cccc}
-1 & 1 & -1 & -1 \\
-1 & 1 & -1 & -1 \\
-1 & 1 & -1 & -1 \\
-1 & 1 & -1 & -1 \\
1 & -1 & -1 & -1 \\
-1 & -1 & 1 & -1 \\
-1 & -1 & 1 & -1
\end{array}\right]\left[\begin{array}{cccc}
-1 & -1 & -1 & -1 \\
-1 & -1 & 1 & -1 \\
-1 & -1 & 1 & -1 \\
-1 & -1 & -1 & 1 \\
-1 & -1 & 1 & -1 \\
-1 & -1 & 1 & -1 \\
-1 & -1 & -1 & 1
\end{array}\right]\left[\begin{array}{cccc}
1 & -1 & -1 & -1 \\
-1 & 1 & -1 & -1 \\
1 & -1 & -1 & -1 \\
-1 & 1 & -1 & -1 \\
1 & -1 & -1 & -1 \\
1 & -1 & -1 & -1 \\
-1 & -1 & 1 & -1
\end{array}\right]\left[\begin{array}{cccc}
-1 & -1 & 1 & -1 \\
-1 & -1 & -1 & 1 \\
-1 & 1 & -1 & -1 \\
-1 & -1 & 1 & -1 \\
-1 & -1 & 1 & -1 \\
-1 & -1 & 1 & -1 \\
-1 & -1 & -1 & 1
\end{array}\right]} \\
& {\left[\begin{array}{cccc}
-1 & -1 & -1 & 1 \\
-1 & -1 & 1 & -1 \\
-1 & -1 & 1 & -1 \\
-1 & -1 & -1 & 1 \\
-1 & -1 & -1 & 1 \\
-1 & -1 & -1 & 1 \\
-1 & 1 & -1 & -1
\end{array}\right]\left[\begin{array}{cccc}
-1 & 1 & -1 & -1 \\
-1 & 1 & -1 & -1 \\
-1 & -1 & 1 & -1 \\
-1 & -1 & 1 & -1 \\
-1 & 1 & -1 & -1 \\
-1 & 1 & -1 & -1 \\
-1 & -1 & 1 & -1
\end{array}\right]}
\end{aligned}
$$

\subsection{Network Creation and Simulation Learning}

Discrete Hopfield neural network was created by the neural network toolbox function newhop in MATLAB. The ideal cement pavement performance grade evaluation index matrix and the six unclassified cement pavement performance evaluation index matrices were input to the DHNN in the programmed MATLAB program. The classification of cement pavement performance on the test section was obtained through a certain number of simulation studies. The results are as follows:

$$
\begin{aligned}
& {\left[\begin{array}{rrrr}
-1 & 1 & -1 & -1 \\
-1 & 1 & -1 & -1 \\
-1 & 1 & -1 & -1 \\
-1 & 1 & -1 & -1 \\
-1 & 1 & -1 & -1 \\
-1 & 1 & -1 & -1 \\
-1 & 1 & -1 & -1
\end{array}\right]\left[\begin{array}{llll}
-1 & -1 & 1 & -1 \\
-1 & -1 & 1 & -1 \\
-1 & -1 & 1 & -1 \\
-1 & -1 & 1 & -1 \\
-1 & -1 & 1 & -1 \\
-1 & -1 & 1 & -1 \\
-1 & -1 & 1 & -1
\end{array}\right]} \\
& {\left[\begin{array}{rrrrr}
-1 & -1 & -1 & 1 \\
-1 & -1 & -1 & 1 \\
-1 & -1 & -1 & 1 \\
-1 & -1 & -1 & 1 \\
-1 & -1 & -1 & 1 \\
-1 & -1 & -1 & 1 \\
-1 & 1 & -1 & -1 \\
-1 & 1 & -1 & -1 \\
-1 & 1 & -1 & -1 \\
-1 & 1 & -1 & -1 \\
-1 & 1 & -1 & -1 \\
-1 & 1 & -1 & -1 \\
-1 & 1 & -1 & -1
\end{array}\right]\left[\begin{array}{ll}
-1 \\
-1
\end{array}\right]}
\end{aligned}
$$

The performance evaluation grades of the six cement pavements excellent, good, medium, poor. The evaluation results of the DHNN method are compared with the evaluation results of the fuzzy complex matter method ${ }^{[5]}$ and the nonlinear fuzzy method ${ }^{[6]}$. The comparison results are as follows:

Table 3. Pavement performance grading of results

\begin{tabular}{cccc}
\hline Method & $\begin{array}{c}\text { DHNN } \\
\text { method }\end{array}$ & $\begin{array}{c}\text { Fuzzy complex } \\
\text { element method }\end{array}$ & $\begin{array}{c}\text { Nonlinear } \\
\text { fuzzy method }\end{array}$ \\
\hline 1 & Good & Good & Good \\
\hline
\end{tabular}

\begin{tabular}{cccc}
\hline 2 & Medium & Poor & Medium \\
3 & Excellent & Excellent & Excellent \\
4 & Medium & Medium & Poor \\
5 & Poor & Poor & Poor \\
6 & Good & Medium & Good \\
\hline
\end{tabular}

It can be seen from Table 3 that the evaluation results of the discrete Hopfield neural network are basically consistent with the evaluation results of the fuzzy complex matter method and the nonlinear fuzzy method. The evaluation results of the cement pavement performances of B, D and F are slightly different. However, at most one 
evaluation level is different. There are no cases where the evaluation results of the three methods are completely different. The reason why the evaluation results are different is that the partial evaluation index values of the test sections are located near the threshold values of the adjacent two levels. Therefore, the DHNN-based cement pavement performance evaluation method is theoretically reliable.

\section{Conclusion}

In this paper, the discrete Hopfield neural network constructed by MATLAB is used to evaluate the performance of the cement pavement on the test section. The following conclusions are obtained.

1, This paper enriches the indicators in the Code in the selection of cement pavement performance evaluation indicators. Comprehensive evaluation was carried out using seven optimization indicators reflecting the pavement performance and pavement structure in the relevant literature. This is significance for the comprehensive and accurate evaluation of cement pavement performance.

2, The cement pavement performance evaluation method based on discrete Hopfield neural network is relatively simple compared with traditional methods such as BP neural network and radial basis neural network. The function of the MATLAB neural network toolbox is directly used in this method, which doesn't need a large number of training samples. This method has a strong objectivity, which is conducive to saving the cost of the test. The DHNN method is similar to the fuzzy composite matter element method and the nonlinear fuzzy method, which proves the accuracy and scientific of the evaluation results.

3, The cement pavement performance evaluation method based on discrete Hopfield neural network is a comprehensive evaluation method. With the in-depth study of cement pavement performance evaluation indicators, the evaluation indicators will be more comprehensive and the grading standards under each evaluation index are more accurate (the more accurate the equilibrium point of the discrete Hopfield neural network). DHNN's evaluation method is equally applicable and the evaluation results are more accurate. The grade of road performance evaluation is more adaptive to the level of maintenance measures taken.

\section{References}

1. JTJ 073.1-2001 Highway Cement Concrete Pavement Maintenance Technical Specification [S]. Beijing: China Communications Press, 2007: 13-16

2. JTG H20-2007 Highway Technical Status Evaluation Standard [S]. Beijing: China Communications Press, 2007: 21-27

3. Zeng QX. (2008) Research on Performance Evaluation and Prediction of Cement Pavement Based on Support Vector Machine [D]. Changsha University of Science and Technology
4. Chen XY, Li YB. (2014) Evaluation model of cement concrete pavement performance based on fuzzy stratification comprehensive method $[\mathrm{J}]$. China Foreign Highway, 34(05): 66-70.

5. Tian XG, Lin D, Wu SD. (2010) Evaluation of cement pavement performance based on fuzzy composite matter element method[J]. Journal of Traffic and Transportation Engineering, 10(02):26-29+35.

6. Yan KZ, Zhou ZX. (2013) Evaluation of cement pavement performance based on nonlinear fuzzy method[J]. Journal of Zhejiang University (Engineering Science), 47(08):1379-1383+1392.

7. Kaslik, E., \& Sivasundaram, S. (2011) Impulsive hybrid discrete-time Hopfield neural networks with delays and multistability analysis. Neural Networks, 24(4), 370-377. 